\title{
Division protein interaction web: identification of a phylogenetically conserved common interactome between Streptococcus pneumoniae and Escherichia coli
}

Correspondence

Patrizia Ghelardini

ghelardini@bio.uniroma2.it

Received 20 March 2008

Revised 30 May 2008

Accepted 12 June 2008

\author{
Silvia Maggi, ${ }^{1}$ Orietta Massidda, ${ }^{3}$ Giuseppe Luzi, ${ }^{2}$ Daniela Fadda, ${ }^{3}$ \\ Luciano Paolozzi ${ }^{1}$ and Patrizia Ghelardini $i^{1,4}$ \\ ${ }^{1}$ Dipartimento di Biologia, Università Tor Vergata, Roma, Italy \\ ${ }^{2}$ Dipartimento di Medicina Interna, Facoltà di Medicina, Università La Sapienza, Roma, Italy \\ ${ }^{3}$ Dipartimento di Scienze e Tecnologie Biomediche, Sez. Microbiologia Medica, Cagliari, Italy \\ ${ }^{4}$ Istituto di Biologia e Patologia Molecolare del CNR, Roma, Italy
}

\section{INTRODUCTION}

The division machinery of Eubacteria is made through the formation of a macromolecular complex containing at least twelve proteins, which assemble with a defined dependence hierarchy at the site of division. The organization of the genes coding for division proteins is generally conserved, and many different bacteria, including Gram-negative and Gram-positive rods and cocci, possess a chromosomal region similar to that found at the 2 min region of the Escherichia coli chromosome (Yura et al., 1992; Ayala et al., 1994). This region contains many genes whose products are involved in division and cell wall biosynthesis and is therefore known as the $d c w$ (division and cell wall) cluster (Ayala et al., 1994; Vicente \& Errington, 1996). This cluster, which is very similar in organization in many Gramnegative bacteria, shows various differences in Grampositive bacteria, particularly in cocci (Tamames et al., 2001).
Despite the differences in $d c w$ cluster organization, the major proteins involved in the division process appear to be conserved. In particular, at least eight proteins are present in almost all cell-wall-containing Eubacteria - FtsZ, FtsA, FtsK, FtsQ/DivIB, FtsB/DivIC, FtsL, FtsW and FtsI although additional proteins may also be present, depending on the bacterial species (Margolin, 2000; Errington et al., 2003; Harry et al., 2006).

It has been shown that in many species, the cell division proteins, organized by the tubulin-like FtsZ, assemble at the division site to form a molecular division machinery, the septosome or divisome. FtsZ, in fact, directs the cytokinesis process, constituting a scaffold for the other division proteins, which, as determined for the bestcharacterized rod-shaped model organisms E. coli and Bacillus subtilis, are recruited hierarchically at the mid-cell to form the septal machinery (Errington et al., 2003; Margolin, 2003; Weiss, 2004; Goehring \& Beckwith, 2005; Harry et al., 2006; Vicente et al., 2006; Erickson, 2007). As proposed recently, the assembly of the divisome components 
is a multistage process, which proceeds in a concerted way (Goehring et al., 2006; Vicente \& Rico, 2006).

In E. coli, the early event is marked by the interactions of FtsZ with FtsA and ZipA, which assemble in the cytoplasmic membrane (for review see Vicente et al., 2006). After the addition of FtsK, which completes the formation of the cytoplasmic ring, a periplasmic connector formed by the complex FtsQ-FtsB-FtsL is added (Buddelmeijer \& Beckwith, 2004), followed by the proteins involved in manufacturing the septal peptidoglycan, FtsW and FtsI. Finally, FtsN is recruited to the septosome (Vicente et al., 2006).

Despite the differences observed in some aspects of the division mechanism in different bacteria, one common thread is the role of FtsZ as a cell division organizer. Moreover, the similar conservation of many division proteins suggests that they should all be recruited at the Z-ring by a common mechanism.

Streptococcus pneumoniae is a developing model for studying cell division in Gram-positive pathogens. Since the dcw cluster was identified (Massidda et al., 1998), considerable progress has been made in understanding the basic mechanism of septum formation in this Grampositive coccus. In particular, a number of cell division proteins have now been identified in S. pneumoniae and shown to localize at the mid-cell to form the septal machinery, consistent with what is known for the rodshaped model bacteria (Errington et al., 2003; Weiss, 2004; Goehring \& Beckwith, 2005; Vicente et al., 2006). These proteins include the cell division initiator proteins, such as FtsZ and FtsA, required at the early stages of the process (Massidda et al., 1998; Morlot et al., 2003; Lara et al., 2005), some of the later players FtsQ/DivIB, FtsB/DivIC, FtsL, FtsW, PBP2x and PBP1A (Massidda et al., 1998; Morlot et al., 2003, 2004; Noirclerc-Savoye et al., 2005; Zapun et al., 2008), which constitute the septal markers in $S$. pneumoniae cells, and most recently DivIVA, which appears to be required for septum completion and pole maturation (Fadda et al., 2007).

Despite this progress many aspects of cell division in $S$. pneumoniae still remain to be clarified, in particular the sequence of the events necessary for septum formation, including the order of recruitment of the proteins to the cell division site. In addition, very little is known about the complex pattern of interactions among the different players that constitute the divisome.

In model organisms, particularly E. coli, the progression of knowledge has been achieved through the establishment of the requirement of a protein for the sequential localization of the others that follow. In S. pneumoniae, the unavailability of specific thermosensitive or conditional division mutants has so far limited the possibility of establishing precisely the hierarchical order of recruitment, if any, and the interdependence of the division proteins at the midcell, Moreover, although interaction between the strep- tococcal FtsA and FtsZ has been detected (Lara et al., 2005) and biochemical evidence for a trimeric complex, formed by FtsQ/DivIB, FtsL and FtsB/DivIC, has been reported (Noirclerc-Savoye et al., 2005), no other information is available on protein-protein interaction between the other players.

To clarify the different and common pathways of the division mechanism in S. pneumoniae, we studied the division protein interaction network, using a bacterial twohybrid assay, applied previously to study protein-protein interaction among the cell division players in E. coli (Di Lallo et al., 2003) and, more recently, to identify the interactive partners of the S. pneumoniae DivIVA protein (Fadda et al., 2007). Moreover, we compared the streptococcal division interaction web with that of $E$. coli and found that a significant number of the interactions were conserved. Consistently, several cross-interactions between S. pneumoniae proteins and the corresponding $E$. coli orthologues were observed. Taken together, these results indicate the presence of a minimum division interactome in common between these two, phylogenetically distinct, micro-organisms.

\section{METHODS}

Bacterial strains and growth conditions. The S. pneumoniae $\mathrm{Rx} 1$ strain, a non-encapsulated well-characterized laboratory strain, was used as a source of DNA, as described previously (Fadda et al., 2007). E. coli K-12 and derivatives, used and generated in this work, are listed in Table 1. Luria-Bertani (LB) broth or agar was used for bacterial culture and plating and SM (salt solution) was used for bacterial dilutions as described by Miller (1972). The following antibiotics (Sigma) were added to the culture media when required: ampicillin $\left(50 \mu \mathrm{g} \mathrm{ml}^{-1}\right)$, chloramphenicol $\left(34 \mu \mathrm{g} \mathrm{ml}^{-1}\right)$ and kanamycin $\left(30 \mu \mathrm{g} \mathrm{ml}^{-1}\right)$.

DNA manipulation. Standard procedures were used for small-scale plasmid preparations, endonuclease digestion, ligation, agarose gel electrophoresis, elution of DNA fragments from agarose and bacterial transformation (Sambrook et al., 1989). PCR was carried out using the Taq DNA polymerase kit (Promega), according to the recommendations of the manufacturer. Synthetic oligonucleotides for S. pneumoniae and for E. coli, used for gene amplification in the construction of plasmids for the two-hybrid assay, are reported in Fadda et al. (2007) and D'Ulisse et al. (2007), respectively. The upstream and downstream primers, containing the restriction sites for SalI and BamHI (underlined), for amplification of $p b p 2 B$ were spnSF $b p 2 B$ (5'-GCGTCGACCATGAGAAAATTTAACAGCCATTC$\left.3^{\prime}\right)$ and spnBR $b p 2 B$ (5'-CGCGGATCCGCAATAGGTGTTGGATAAAGCA- $3^{\prime}$ ); the start and stop codons, respectively, are indicated in bold.

Plasmids construction and bacterial two-hybrid assay. Recombinant plasmids (listed in Table 1) were constructed by cloning the entire genes of interest into the Sall and BamHI restriction sites of $\mathrm{pcI}_{\mathrm{P} 22}$ and $\mathrm{pcI}_{434}$ vectors (Di Lallo et al., 2001). The DNA of the gene of interest was obtained by PCR amplification using specific oligonucleotides carrying compatible restriction sites at the ends. All the different combinations of recombinant plasmids coding for the chimeric repressors, obtained as described above, were cotransformed into the recipient E. coli strain R721, carrying the 434/ P22 chimeric operator. $\beta$-Galactosidase activity was assayed as described by Miller (1972). Bacterial cultures were grown at $34{ }^{\circ} \mathrm{C}$ 
Table 1. Bacterial strains and plasmids used in the study

\begin{tabular}{|c|c|c|}
\hline Strain or plasmid & Relevant genotype & Source \\
\hline \multicolumn{3}{|l|}{ E. coli strains } \\
\hline $71 / 18$ & supE thy $\Delta($ lac pro $A B) / \mathrm{F}^{\prime}\left(\right.$ proAB $B^{+}$lacI $I^{\mathrm{q}}$ lacZ $\left.\Delta \mathrm{M} 15\right)$ & Lab collection \\
\hline $\mathrm{DH} 5 \alpha$ & supE44 $\Delta$ lacU169 hsdR17 recA1 endA1 gyrA96 thil relA1 & Lab collection \\
\hline R721 & $71 / 18 \mathrm{glpT}:: \mathrm{OP}_{434 / \mathrm{P} 22} l a c Z$ & Di Lallo et al. (2001) \\
\hline \multicolumn{3}{|l|}{ Plasmids } \\
\hline pTTQ18 $g f p$ & pTTQ18 derivative harbouring $g f p$ gene & D’Ulisse et al. (2007) \\
\hline pBAD33 gst & pBAD33 derivative harbouring gst gene & D’Ulisse et al. (2007) \\
\hline pTTQ18 $g f p$-spnftsZ & pTTQ18 $g f p$ derivative harbouring $f t s Z$ gene of $S$. pneumoniae & Fadda et al. (2007) \\
\hline pTTQ18 $g f p$-spnfts $W$ & pTTQ18 $g f p$ derivative harbouring $f t s W$ of $S$. pneumoniae & This work \\
\hline pTTQ18 $g f p$-spn $p b p 2 x$ & pTTQ18 $g f p$ derivative harbouring $p b p 2 x$ of $S$. pneumoniae & This work \\
\hline pTTQ18 gfp-spnftsA & pTTQ18 $g f p$ derivative harbouring $f t s A$ of $S$. pneumoniae & This work \\
\hline pBAD33 gst-spndivIC & pBAD33 gst derivative harbouring divIC of $S$. pneumoniae & This work \\
\hline pBAD33 gst-spnftW & pBAD33 gst derivative harbouring ftsW of $S$. pneumoniae & This work \\
\hline pBAD33 gst-spnftsK & pBAD33 gst derivative harbouring ftsK of $S$. pneumoniae & This work \\
\hline pBAD33 gst-spnftsL & pBAD33 gst derivative harbouring $f t s L$ of $S$. pneumoniae & This work \\
\hline $\mathrm{pcI}_{\mathrm{P} 22}$-ecfts $Z$ & $\mathrm{pcI}_{\mathrm{P} 22}$ derivative harbouring $E$. coli fts $Z$ & Di Lallo et al. (2003) \\
\hline $\mathrm{pcI}_{434}$-ecfts $Z$ & $\mathrm{pcI}_{434}$ derivative harbouring $E$. coli fts $Z$ & Di Lallo et al. (2003) \\
\hline $\mathrm{pcI}_{\mathrm{P} 22}$-ecftsA & $\mathrm{pcI}_{\mathrm{P} 22}$ derivative harbouring $E$. coli ftsA & Di Lallo et al. (2003) \\
\hline $\mathrm{pcI}_{434}$-ecftsA & $\mathrm{pcI}_{434}$ derivative harbouring $E$. coli fts $A$ & Di Lallo et al. (2003) \\
\hline $\mathrm{pcI}_{\mathrm{P} 22}$-ecftsK & pcI $_{\mathrm{P} 22}$ derivative harbouring $E$. coli ftsK & Di Lallo et al. (2003) \\
\hline $\mathrm{pcI}_{434}$-ecftsK & $\mathrm{pcI}_{434}$ derivative harbouring $E$. coli ftsK & Di Lallo et al. (2003) \\
\hline $\mathrm{pcI}_{\mathrm{P} 22}$-ecftsQ & pcI $_{\mathrm{P} 22}$ derivative harbouring $E$. coli fts $Q$ & Di Lallo et al. (2003) \\
\hline $\mathrm{pcI}_{434}$-ecftsQ & $\mathrm{pcI}_{434}$ derivative harbouring E. coli ftsQ & Di Lallo et al. (2003) \\
\hline $\mathrm{pcI}_{\mathrm{P} 22}$-ecftsL & $\mathrm{pcI}_{\mathrm{P} 22}$ derivative harbouring $E$. coli ftsL & Di Lallo et al. (2003) \\
\hline $\mathrm{pcI}_{434}$-ecftsL & $\mathrm{pcI}_{434}$ derivative harbouring E. coli ftsL & Di Lallo et al. (2003) \\
\hline $\mathrm{pcI}_{\mathrm{P} 22}$-ecftsW & pcI $_{\mathrm{P} 22}$ derivative harbouring $E$. coli ftsW & Di Lallo et al. (2003) \\
\hline $\mathrm{pcI}_{434}$-ecftsW & $\mathrm{pcI}_{434}$ derivative harbouring $E$. coli ftsW & Di Lallo et al. (2003) \\
\hline $\mathrm{pcI}_{\mathrm{P} 22-\mathrm{ec} z a p A}$ & pcI $_{\mathrm{P} 22}$ derivative harbouring E. coli zapA & Di Lallo et al. (2003) \\
\hline $\mathrm{pcI}_{434}-\mathrm{ec} z a p A$ & $\mathrm{pcI}_{434}$ derivative harbouring E. coli zapA & Di Lallo et al. (2003) \\
\hline $\mathrm{pcI}_{434}$-ecftsB & $\mathrm{pcI}_{434}$ derivative harbouring $E$. coli ftsB & D’Ulisse et al. (2007) \\
\hline $\mathrm{pcI}_{\mathrm{P} 22}-\mathrm{ec} f t s B$ & $\mathrm{pcI}_{\mathrm{P} 22}$ derivative harbouring $E$. coli ftsB & D’Ulisse et al. (2007) \\
\hline $\mathrm{pcI}_{\mathrm{P} 22}-\mathrm{spn} f t s Z$ & $\mathrm{pcI}_{\mathrm{P} 22}$ derivative harbouring $S$. pneumoniae fts $Z$ & Fadda et al. (2007) \\
\hline $\mathrm{pcI}_{434}$-spnfts $Z$ & $\mathrm{pcI}_{434}$ derivative harbouring $S$. pneumoniae fts $Z$ & Fadda et al. (2007) \\
\hline $\mathrm{pcI}_{\mathrm{P} 22}-\mathrm{spn} f t s A$ & $\mathrm{pcI}_{\mathrm{P} 22}$ derivative harbouring $S$. pneumoniae fts $A$ & Fadda et al. (2007) \\
\hline $\mathrm{pcI}_{434}$-spnftsA & $\mathrm{pcI}_{434}$ derivative harbouring $S$. pneumoniae ftsA & Fadda et al. (2007) \\
\hline $\mathrm{pcI}_{434}$-spnzapA & $\mathrm{pcI}_{434}$ derivative harbouring $S$. pneumoniae zapA & Fadda et al. (2007) \\
\hline $\mathrm{pcI}_{\mathrm{P} 22}$-spnzapA & pcI $_{\mathrm{P} 22}$ derivative harbouring $S$. pneumoniae zapA & Fadda et al. (2007) \\
\hline $\mathrm{pcI}_{434}$-spnftsK & $\mathrm{pcI}_{434}$ derivative harbouring $S$. pneumoniae ftsK & Fadda et al. (2007) \\
\hline $\mathrm{pcI}_{\mathrm{P} 22}$-spnftsK & $\mathrm{pcI}_{434}$ derivative harbouring $S$. pneumoniae ftsK & Fadda et al. (2007) \\
\hline $\mathrm{pcI}_{\mathrm{P} 22}$-spndivIB & pcI $_{\mathrm{P} 22}$ derivative harbouring $S$. pneumoniae divIB & Fadda et al. (2007) \\
\hline $\mathrm{pcI}_{434}$-spndivIB & $\mathrm{pcI}_{434}$ derivative harbouring $S$. pneumoniae divIB & Fadda et al. (2007) \\
\hline $\mathrm{pcI}_{\mathrm{P} 22}$-spndivIC & $\mathrm{pcI}_{\mathrm{P} 22}$ derivative harbouring $S$. pneumoniae divIC & Fadda et al. (2007) \\
\hline $\mathrm{pcI}_{434}$-spndivIC & $\mathrm{pcI}_{434}$ derivative harbouring $S$. pneumoniae divIC & Fadda et al. (2007) \\
\hline $\mathrm{pcI}_{\mathrm{P} 22}-\mathrm{spn} f t s L$ & $\mathrm{pcI}_{\mathrm{P} 22}$ derivative harbouring $S$. pneumoniae ftsL & Fadda et al. (2007) \\
\hline $\mathrm{pcI}_{434}$-spnftsL & $\mathrm{pcI}_{434}$ derivative harbouring $S$. pneumoniae ftsL & Fadda et al. (2007) \\
\hline $\mathrm{pcI}_{\mathrm{P} 22}$-spnftsW & $\mathrm{pcI}_{\mathrm{P} 22}$ derivative harbouring $S$. pneumoniae ftsW & Fadda et al. (2007) \\
\hline $\mathrm{pcI}_{434}$-spnftsW & $\mathrm{pcI}_{434}$ derivative harbouring $S$. pneumoniae ftsW & Fadda et al. (2007) \\
\hline $\mathrm{pcI}_{\mathrm{P} 22-\mathrm{spn} p b p 2 X}$ & $\mathrm{pcI}_{\mathrm{P} 22}$ derivative harbouring $S$. pneumoniae $p b p 2 X$ & Fadda et al. (2007) \\
\hline $\mathrm{pcI}_{434}$-spn $p b p 2 X$ & $\mathrm{pcI}_{434}$ derivative harbouring $S$. pneumoniae $p b p 2 X$ & Fadda et al. (2007) \\
\hline pcI $\mathrm{I}_{22}-\mathrm{spn} p b p 1 A$ & pcI $_{\mathrm{P} 22}$ derivative harbouring $S$. pneumoniae $p b p 1 A$ & Fadda et al. (2007) \\
\hline $\mathrm{pcI}_{434}$-spn $p b p 1 A$ & $\mathrm{pcI}_{434}$ derivative harbouring $S$. pneumoniae $p b p 1 A$ & Fadda et al. (2007) \\
\hline $\mathrm{pcI}_{\mathrm{P} 22}-\mathrm{spn} p b p 2 B$ & pcI $_{\mathrm{P} 22}$ derivative harbouring $S$. pneumoniae $p b p 2 B$ & This work \\
\hline $\mathrm{pcI}_{434}$-spn $p b p 2 B$ & $\mathrm{pcI}_{434}$ derivative harbouring $S$. pneumoniae $p b p 2 B$ & This work \\
\hline
\end{tabular}


in LB medium supplemented with $0.1 \mathrm{mM}$ IPTG to $\mathrm{OD}_{600} 0.5$, and the residual $\beta$-galactosidase activity was evaluated for each strain. $E$. coli R721 without plasmids and E. coli R721 with plasmids pcI $_{434} 434$ and $\mathrm{pcIP}_{22} 434$ were used as negative and positive controls, respectively. Residual $\beta$-galactosidase activity of less than $50 \%$ was judged to indicate that there was repression and hence a proteinprotein interaction, whereas when the activity was greater than $50 \%$, the interaction was uncertain or null. The use of these values has been discussed previously (Di Lallo et al., 2003; D’Ulisse et al., 2007; Fadda et al., 2007).

Construction of GFP and GST derivatives for co-immunoprecipitation studies. The division proteins fused with GFP (green fluorescent protein of Aequorea victoria) were obtained by in-frame cloning of the PCR-produced DNA fragments of the corresponding gene into PstI and HindIII sites downstream of the $g f p$ gene previously inserted into the EcoRI and PstI sites in the plasmid pTTQ18 to give the plasmid pTTQ18-gfp (D'Ulisse et al., 2007; Fadda et al., 2007; Table 1). Analogously, the GST (glutathione $S$-transferase of Schistosoma japonicum) derivatives were obtained by cloning the gene of interest into SalI and HindIII sites downstream of the gst gene inserted into the SacI and SalI sites of the plasmid pBAD33 to give the plasmid pBAD33-gst (D’Ulisse et al., 2007; Table 1).

Co-immunoprecipitation experiments. To prepare the extracts for co-immunoprecipitation experiments, cultures of E. coli DH5 $\alpha$, harbouring the recombinant plasmids containing the genes of interest fused in-frame to GST and GFP, were grown in LB supplemented with the appropriate antibiotics to $\mathrm{OD}_{600} 0.3-0.4$. The expression of the tagged genes was induced by adding $0.1 \mathrm{mM}$ IPTG (for the GFPfused genes) and $0.2 \%$ arabinose (for the GST-fused gene) and the culture was grown for $4 \mathrm{~h}$. The culture was then centrifuged, washed and resuspended $(1 / 300, \mathrm{v} / \mathrm{v})$ in lysis buffer $(1 \mathrm{mM}$ EDTA pH 8 , $25 \mathrm{mM}$ HEPES pH 7.6, $0.1 \mathrm{mg}$ lysozyme $\mathrm{ml}^{-1}$ ), cooled on ice for $30 \mathrm{~min}$, and lysed by freezing and thawing. The protein concentration, determined by the Bradford method after cell lysis, was adjusted to $10 \mathrm{mg} \mathrm{ml}^{-1}$ and $1 \mathrm{ml}$ aliquots of lysed samples were centrifuged at $50000 \boldsymbol{g}$ for $45 \mathrm{~min}$. Soluble fractions were obtained by recovering the supernatant, and membrane fractions were prepared as described by Buddelmeijer \& Beckwith (2004). Immunoprecipitation experiments were similar to those described by Duong \& Wickner (1997). The beads (Protein A-Sepharose) were divided into aliquots of 50$100 \mu \mathrm{l}$ in microcentrifuge tubes; $10 \mu \mathrm{l}$ anti-GFP $(1: 100)$ (Santa Cruz Biotechnology) or anti-GST $(1: 100)$ (Santa Cruz Biotechnology) mouse monoclonal antibodies were added to each tube. The samples were incubated overnight at $4{ }^{\circ} \mathrm{C}$ with gentle mixing on a suitable shaker. After centrifugation, samples were washed with $1 \mathrm{ml}$ washing buffer (20 mM HEPES buffer, pH 7.5, containing $150 \mathrm{mM} \mathrm{NaCl}$, $0.1 \%$ Triton X-100 and $10 \%, \mathrm{v} / \mathrm{v}$, glycerol), then $0.1-1 \mathrm{ml}$ cell lysate was added to each tube. Samples were incubated from $90 \mathrm{~min}$ to overnight at $4{ }^{\circ} \mathrm{C}$, with gentle mixing on a suitable shaker. Immunoprecipitated complexes were collected by centrifugation at $3000 \mathrm{~g}$ for $2 \mathrm{~min}$ at $4{ }^{\circ} \mathrm{C}$.

Western blotting. Electrophoresis and immunoblotting were performed as described by Laemmli (1970) and Towbin et al. (1979), respectively. The nitrocellulose membranes were probed with anti-GST antibodies (Santa Cruz Biotechnology) $(1: 2000)$ or with mouse anti-GFP monoclonal antibodies $(1: 1000)$ (Santa Cruz Biotechnology), depending on the specific GFP- or GST-fused protein used in the experiment, and detected with the ECF fluorescence-based detection system (Amersham, Pharmacia). The specific polyclonal antibodies raised against $S$. pneumoniae division proteins DivIB, FtsL, FtsK, FtsW, PBP2x, PBP1A and PBP2B used in Western blot experiments were generously supplied by A. Zapun and T. Vernet of the Institut de Biologie Structurale, CNRS, Grenoble, France.

\section{RESULTS}

\section{Identification of S. pneumoniae division protein interaction network}

A bacterial two-hybrid assay (THA), developed previously in our laboratory and used successfully to describe the $E$. coli division protein interaction web (Di Lallo et al., 2003), was applied to dissect the analogous pathway in $S$. pneumoniae. In this assay, the interaction between two proteins, encoded by the corresponding genes cloned in the two recombinant plasmids, is tested by their ability to form a chimeric lambdoid repressor. When the gene products interact they shut down the expression of a chromosomal copy of the reporter gene, lac $Z$, whose synthesis is governed by a hybrid promoter/operator region, recognized by the chimeric repressor consisting of the $\mathrm{N}$-terminal domain of the lytic phage repressors from either of two lambdoid phages 434 and P22 fused in-frame with the pair of division proteins whose interaction is under investigation (Di Lallo et al., 2001). In particular, we tested the cell division proteins that are known or are thought to participate in divisome formation in $S$. pneumoniae, like FtsZ, FtsA, ZapA, FtsK, FtsQ/DivIVB, FtsB/DivIC, FtsL, FtsW, PBP2x and PBP1A. In addition, we tested PBP2B, an essential monofunctional transpeptidase, the gene of which in S. pneumoniae is located in the $d c w$ cluster with ftsA and ftsZ (Massidda et al., 1998).

Since $S$. pneumoniae fusion proteins were expressed in a heterologous system, we first tested the correct expression of those proteins for which specific antibodies were available, namely FtsZ, FtsA, FtsL, FtsQ/DivIB, PBP1A, PBP2B and PBP2x, by Western blot experiments (data not shown). Although the Western blot results cannot be generalized for the other S. pneumoniae proteins for which antibodies were not available, since all the proteins tested interact with at least one division partner, as shown in Table 2, such interaction should account for correct expression of the recombinant protein.

THA results are presented as the ratio between the $\beta$ galactosidase units (Miller units) produced by the strain harbouring the two recombinant plasmids and that of the same strain with plasmid vectors alone, as a control. We interpret $\beta$-galactosidase percentages lower than $50 \%$ to represent an interaction between the two proteins under investigation whereas percentages over $50 \%$ indicate a lack of interaction. Values close to $50 \%$ could represent weak or transient interactions. The rationale for this cut-off is reported in previous work (Di Lallo et al., 2003; D'Ulisse et al., 2007; Fadda et al., 2007).

The results reported in Table 2 highlight that, out of 66 combinations among the $S$. pneumoniae cell division proteins tested, 37 show homo or hetero-dimerizations, while 29 did not interact, either with themselves or with each other. Of the streptococcal cell division proteins shown in Table 2, 18 (i.e. 14 positive interactions and four non-interactions) were consistent with those established 
Table 2. Homo- and hetero-dimerization of 11 S. pneumoniae known or putative cell division proteins

Sixty-six possible combinations of recombinant plasmids were tested to reconstruct a functional 434-P22 chimeric repressor able to bind the chimeric operator $\mathrm{O}-\mathrm{P}_{434 / \mathrm{P} 22}$ of E. coli strain R721, where it controls the expression of the lacZ reporter gene. Each of these plasmids carries the Nterminal domain of the 434 or P22 phage repressor fused in-frame with the coding sequence of one of the 11 streptococcal cell division genes. The effective binding to the operator sequence is deduced by the repression of $\beta$-galactosidase synthesis, evaluated by the ratio between the Miller units of $\beta$-galactosidase produced by strain R721 carrying each pair of plasmids and that of R721 without plasmids (Di Lallo et al., 2003). The assay was performed as described in Methods. The results represent the means of at least five independent determinations, in which standard deviation did not exceed \pm 4 . The residual $\beta$-galactosidase activity of the positive and negative controls was $5 \%$ and $100 \%$, respectively. Values of less than $50 \%$ (in bold) indicate an interaction between the two proteins under investigation. Similar results were obtained when the genes were cloned in the reciprocal vectors (not shown).

\begin{tabular}{|c|c|c|c|c|c|c|c|c|c|c|c|}
\hline $\mathrm{pcI}_{434}$ & FtsA & FtsZ & ZapA & FtsK & $\begin{array}{l}\text { FtsQ/ } \\
\text { DivIB }\end{array}$ & $\begin{array}{l}\text { FtsB/ } \\
\text { DivIC }\end{array}$ & FtsL & FtsW & PBP2x & PBP1A & PBP2B \\
\hline FtsA & 19 & & & & & & & & & & \\
\hline FtsZ & 22 & 18 & & & & & & & & & \\
\hline ZapA & 9 & 5 & 9 & & & & & & & & \\
\hline $\begin{array}{l}\text { FtsQ/ } \\
\text { DivIB }\end{array}$ & 58 & 27 & 5 & 23 & 30 & & & & & & \\
\hline $\begin{array}{l}\text { FtsB/ } \\
\text { DivIC }\end{array}$ & 71 & 16 & 17 & 61 & 81 & 35 & & & & & \\
\hline FtsL & 26 & 17 & 14 & 30 & 15 & 58 & 29 & & & & \\
\hline FtsW & 53 & 31 & 27 & 31 & 40 & 34 & 20 & 55 & & & \\
\hline
\end{tabular}

previously for E. coli, by two different THAs (Di Lallo et al., 2003; Karimova et al., 2005) and co-immunoprecipitation experiments (D'Ulisse et al., 2007). Some of these interactions, like those of the $S$. pneumoniae FtsA with itself and with FtsZ, were expected, confirming and extending our previous results (Lara et al., 2005), by using a yeast two-hybrid system, and thus providing additional reliable positive controls. Moreover, the predicted FtsZ selfinteraction, which could not be determined before, due to the activation of the reporter gene of single transformants producing the FtsZ protein when fused to the GAL4-binding domain (Lara et al., 2005), was detected. Besides FtsA and FtsZ, other self-interacting cell division proteins were FtsQ/DivIB, FtsB/DivIC, PBP2x, FtsK, FtsL and ZapA, consistent with the same behaviour shown by the E. coli counterparts (Di Lallo et al., 2003; P. Ghelardini, unpublished results; see below).

As shown in Table 2, a total of 29 hetero-interactions were detected among $S$. pneumoniae proteins and of these seven, FtsA-FtsK, FtsA-FtsL, FtsZ-FtsW, FtsZ-FtsQ/DivIB, FtsZFtsL, FtsK-FtsW, FtsL-FtsI/PBP2x, have not been reported previously in E. coli (Di Lallo et al., 2003; Karimova et al., 2005), although a positive FtsZ-FtsW interaction was found in Mycobacterium tuberculosis (Datta et al., 2002). Interestingly, it has been suggested recently that binding of FtsZ to the C-tail of FtsW may modulate its interactions with FtsI, regulating septal peptidoglycan biogenesis (Datta et al., 2006). It would be interesting to determine if this is also the case for $S$. pneumoniae, where surprisingly no interaction between FtsW and any of the PBPs tested was detected.

Two other hetero-interactions, FtsZ-FtsB(DivIC) and FtsW-FtsB(DivIC), were identified and seemed to be specific of $S$. pneumoniae (see below).

Among the PBPs, PBP2B was found to interact strongly with FtsZ and ZapA, further supporting the presence of this PBP at the mid-cell, as reported recently (Zapun et al., 2008; D. Fadda \& O. Massidda, unpublished results).

\section{Co-immunoprecipitation of interacting protein pairs identified by THA}

To confirm the results of the THA, co-immunoprecipitation was used. Because of the large number of interactions detected between the proteins pairs examined, only some of them were tested with this additional method. In particular, we chose to confirm those interactions that were shown only in $S$. pneumoniae and not detected previously in E. coli, namely: FtsA-FtsK, FtsA-FtsL, FtsZ-FtsW, FtsZFtsQ/DivIB, FtsZ-FtsL, FtsK-FtsW, FtsL-PBP2x, FtsZFtsB/DivIC and FtsW-FtsB/DivIC.

Since specific antibodies were not available for all these studied proteins, the assays were performed on the division 
proteins fused with tags, i.e. the GFP and/or GST for which commercial antibodies are available and the specificity of the assay has already been demonstrated (D'Ulisse et al., 2007; Fadda et al., 2007).

The results, presented in Fig. 1, show that, as expected from the THA, an interaction was observed for each of the eight pairs of proteins tested. On the other hand, control experiments with non-interacting protein pairs, like FtsLFtsB, FtsK-PBP2x and FtsW-FtsA, did not co-immunoprecipitate (data not shown).

\section{An interaction network genetically conserved in S. pneumoniae and E. coli}

E. coli is the only micro-organism for which the whole interaction web of the divisome proteins has been described (Di Lallo et al., 2003; Karimova et al., 2005; D'Ulisse et al., 2007), and therefore the only possible reference with which to compare the data from S. pneumoniae described above. As noted, these two species share at least nine division proteins and to compare the two interaction webs, we used the data already published on E. coli (Di Lallo et al., 2003) integrated with the data on new interactions detected for E. coli ZapA and FtsB proteins with themselves and with the other E. coli division partners. The results, reported in Table 3 , show that ZapA interacted with itself and with all the other cell division proteins tested except FtsI, while FtsB interacted with itself, ZapA, FtsQ, FtsL and FtsI.

In addition, given the large number of interactions shown by $S$. pneumoniae FtsL ( $\mathrm{FtsL}_{\mathrm{SPN}}$ ), the interaction profile of the FtsL E. coli (FtsL $\mathrm{ECC}_{\mathrm{EC}}$ ) counterpart was re-evaluated. While we found consistent results confirming the heterointeractions already reported (Di Lallo et al., 2003), the ability of FtsL $\mathrm{E}_{\mathrm{EC}}$ to self-interact was also detected, given the $22 \%$ residual $\beta$-galactosidase activity, thus correcting the value reported previously (Di Lallo et al., 2003).

As shown in Fig. 2, in both organisms it is possible to identify a complex interaction network, in which each protein interacts with at least one, but more often with several partners. Although it was clear that in $S$. pneumoniae the interactions are more numerous than
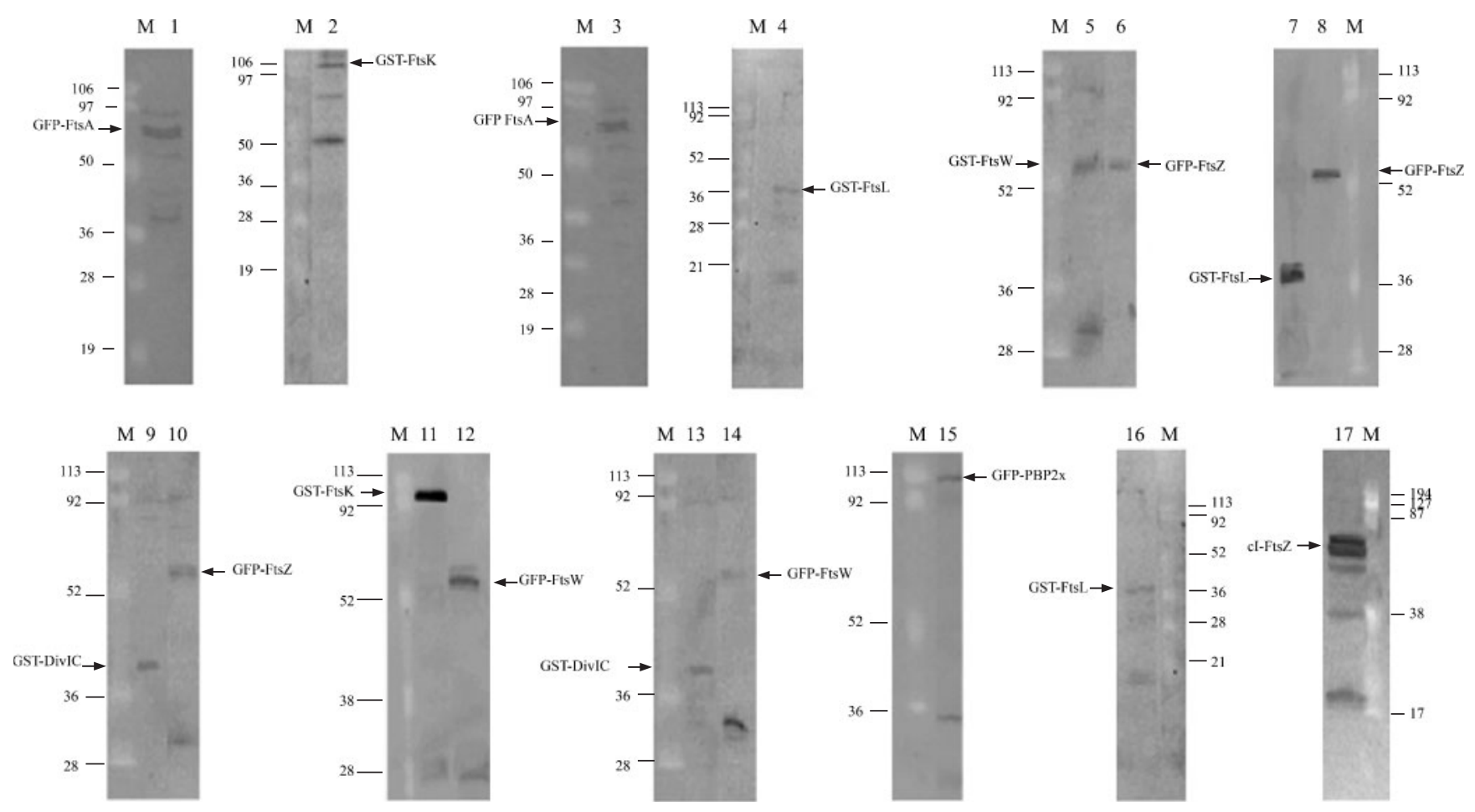

Fig. 1. Co-immunoprecipitation to determine the specificity of $S$. pneumoniae cell division protein interactions. Immunoprecipitation of GFP-FtsA-GST-FtsK (lanes 1-2) and GFP-FtsA-GST-FtsL (lanes 3-4), GST-FtsW-GFP-FtsZ (lanes 5-6), GST-FtsL-GFP-FtsZ (lanes 7-8), GST-DivIC-GFP-FtsZ (lane 9-10), GST-FtsK-GFP-FtsW (lanes 11-12), GST-DivIC-GFP-FtsW (lanes 13-14), and GFP-PBP2x-GST-FtsL (lanes 15-16) complexes is shown. Protein extracts were precipitated with anti-GST (lanes 1, 3, 6, 8, 10,12, 14 and 15) or anti-GFP (lanes 2, 4, 5, 7, 9, 11, 13 and 16) antibodies and detected with anti-GFP or anti-GST antibodies, respectively. In the case of the interaction between DivIB and FtsZ, the extract was precipitated with anti-GFP antibodies (directed to GFP_DivlB) and the co-immunoprecipitate detected with polyclonal anti-FtsZ antibodies (lane 17). Lanes $\mathrm{M}$ contain molecular mass markers. Immunoprecipitation results for non-interacting protein pairs are not shown. 
Table 3. Interactions between the E. coli FtsB and ZapA proteins with themselves and with the other division proteins

The interaction of ZapA with FtsA and FtsZ have been described previously (Di Lallo et al., 2003). The results represent the means of at least five independent determinations, in which standard deviation did not exceed \pm 4 . Values of less than $50 \%$ (in bold) indicate an interaction between the two proteins under investigation, as reported in Methods.

\begin{tabular}{|lccccccccc|}
\hline & FtsA & FtsZ & FtsK & FtsQ & FtsB & FtsL & FtsW & FtsI & ZapA \\
\hline FtsB & 85 & 90 & 67 & $\mathbf{3 6}$ & $\mathbf{2 1}$ & $\mathbf{2 7}$ & 63 & $\mathbf{3 0}$ & \\
ZapA & $\mathbf{2 5}$ & $\mathbf{1 9}$ & $\mathbf{8}$ & $\mathbf{2 9}$ & $\mathbf{5}$ & $\mathbf{6}$ & $\mathbf{8}$ & 85 & $\mathbf{2}$ \\
\hline
\end{tabular}

those in E. coli, $71 \%$ vs $64 \%$, respectively, a closer look revealed a high similarity. Indeed, a large part of the interaction pattern between these two organisms seemed to be conserved. Out of 45 protein pairs tested for each bacterium, 30 show the same behaviour: 23 interacted and seven did not. Notably, all eight homo-interactions were conserved in both organisms. On the other hand, nine interactions appeared specific to $S$. pneumoniae (Fig. 2a), and six to E. coli (Fig. 2b).

Concerning the protein pairs FtsA-FtsQ/DivIB, FtsAFtsW, FtsA-FtsB/DivIC, FtsZ-FtsI (PBP2x/PBP3), ZapA/ FtsI, FtsK-FtsB/DivIC, FtsW-FtsW, no interactions were detected in either organism.

Unexpectedly, the $S$. pneumoniae FtsW did not interact with PBP2x, or any other PBP tested. However, it should be noted that $f t s W$ is absent from the $d c w$ cluster of many Gram-positive organisms, in particularly cocci (Massidda et al., 1998). Furthermore, it has been reported recently that in Staphylococcus aureus the recruitment of PBP2 to the division site is dependent on its substrate (Pinho \& Errington, 2005). If this is also the case for S. pneumoniae PBPs, it could explain the lack of this interaction, although this hypothesis needs to be confirmed.

\section{Heterologous interactions between $E$. coli and S. pneumoniae division proteins}

Heterologous interactions between cell division proteins were first reported by Wang et al. (1997) using a yeast THA. These authors showed that both FtsZ and FtsA of $B$. subtilis were able to interact with FtsZ of E. coli, despite the phylogenetic distance between the two species.

The common pattern of interactions, conserved between two organisms as phylogenetically distant as E. coli and $S$. pneumoniae, suggests that, in this case, the interactions between these proteins (or their domains/structures) could have been maintained over the course of evolution, being fundamental to the division process. It was therefore very interesting to see whether the single members of each pair could cross-interact with the respective orthologous protein, i.e. if a selected $S$. pneumoniae protein would interact with its interactive E. coli counterpart and vice versa. To test this possibility, cross-interactions among heterologous proteins were tested with the THA for all those proteins that showed a conserved interaction profile in the species-specific assay.

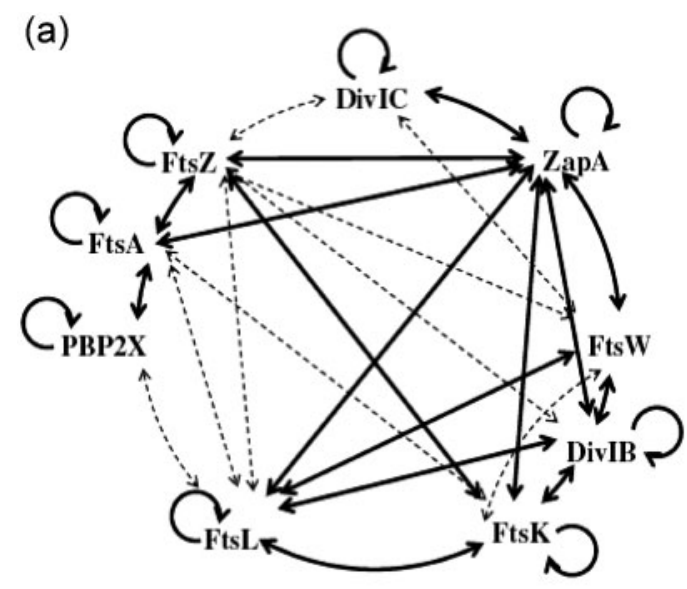

Streptococcus pneumoniae

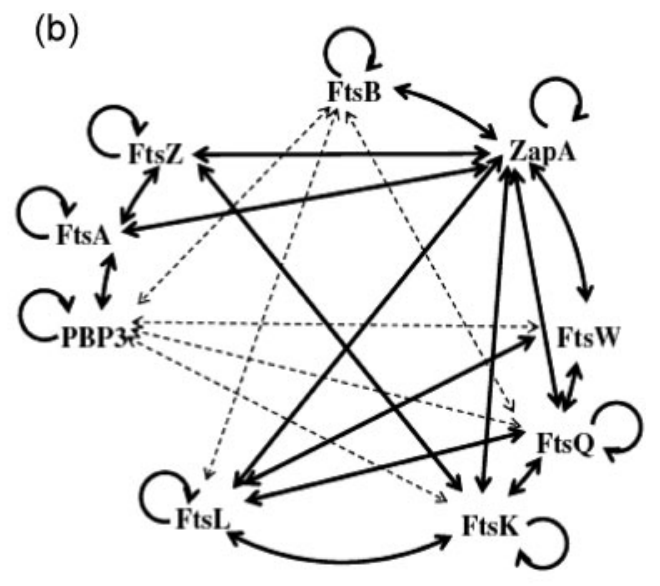

Escherichia coli

Fig. 2. Species-specific interaction profile among the nine cell division proteins common to $S$. pneumoniae and $E$. coli. (a) Interaction web among nine selected S. pneumoniae division proteins, FtsZ, FtsA, ZapA, FtsK, FtsQ/DivlB, FtsB/DivlC, FtsL, FtsW and Ftsl/PBP2x, described in Table 2. (b) Interaction web among the nine E. coli cell division proteins, FtsZ, FtsA, FtsK, FtsQ/DivlB, FtsB/DivlC, FtsL, FtsN, FtsW and Ftsl/PBP3 as described by Di Lallo et al. (2003), supplemented with the results reported in Table 3. Homodimerization is represented by circular arrows. The unbroken arrows indicate conserved interactions, while the dashed arrows indicate species-specific interactions. The double arrows indicate that heterodimerization was studied bi-directionally, with both possible constructions of fusion proteins: 434-X/P22-Y and 434-Y/P22-X. 
The results, presented in Fig. 3, indicate that most of the shared common interactions detected previously, on the basis of the comparison of the two interaction webs, were also conserved in cross-interaction experiments. The crossinteractions, illustrated in the figures, concern the protein pairs FtsZ $\mathrm{EC}_{\mathrm{EC}}-\mathrm{FtsZ}_{\mathrm{SPN}}, \mathrm{FtsA}_{\mathrm{EC}}-\mathrm{FtsZ}_{\mathrm{SPN}}$, Fts $\mathrm{Q}_{\mathrm{EC}}-$ DivIB $_{\mathrm{SPN}}$, $\mathrm{FtsQ}_{\mathrm{EC}}-\mathrm{FtsL}_{\mathrm{SPN}}, \mathrm{FtsL}_{\mathrm{EC}}-\mathrm{FtsL}_{\mathrm{SPN}}, \mathrm{FtsL}_{\mathrm{EC}}-\mathrm{FtsW}_{\mathrm{SPN}}, \mathrm{FtsK}_{\mathrm{EC}}{ }^{-}$ FtsZ $Z_{S P N}, F_{t s K} K_{E C}-D_{i v I B} B_{S P N}, F_{t s K} K_{E C}-F_{s t} L_{S P N}$ and vice versa, with the exception of the pairs FtsK-FtsZ and FtsK-FtsQ. In these cases we observed an interaction only unidirectionally between $\mathrm{FtsK}_{\mathrm{EC}}-\mathrm{FtsZ}_{\mathrm{SPN}}$ and $\mathrm{FtsK}_{\mathrm{EC}}-\mathrm{DivIB}_{\mathrm{SPN}}$; in the opposite orientation, i.e. FtsK $\mathrm{SPN}_{\mathrm{SP}}-\mathrm{FtsZ}_{\mathrm{EC}}$ and $\mathrm{FtsK}_{\mathrm{SPN}}-\mathrm{FtsQ}_{\mathrm{EC}}$, no interactions were detected. Finally, five cross-interactions were observed between $\mathrm{ZapA}_{\mathrm{EC}}$ and the streptococcal proteins $\mathrm{FtsK}_{\mathrm{SPN}}, \mathrm{DivIC}_{\mathrm{SPN}}$, DivIB ${ }_{\text {SPN }}$, $\mathrm{FtsL}_{\mathrm{SPN}}$ and $\mathrm{FtsW}_{\mathrm{SPN}}$. In this case, however, analysis of the reverse interactions was not performed.

\section{DISCUSSION}

In S. pneumoniae the cell division gene organization differs significantly from that of other Eubacteria although the division proteins are phylogenetically conserved (Massidda et al., 1998). In this organism, at least 12 proteins are known or believed to be involved in the process and, among them, nine, FtsZ, FtsA, ZapA, FtsK, FtsQ/DivIB, FtsB/DivIC, FtsL, FtsW and FtsI/PBP2x, are common with both other Gram-positive and Gram-negative bacteria.

A function has been attributed to only a few division proteins. This identification of function is difficult because often the inactivation of the respective genes is not lethal in conditions of overexpression of other division proteins. A common property of all these proteins is their colocalization at the division site. In the last few years, substantial progress has been made in elucidating several aspects of cell division in various bacterial models, such as identification of genes, and structure and activity of the proteins involved in the process, their cytological localization, Z-ring formation, etc. All this knowledge cannot however give us information on the assembly of the division proteins or the regulation and the structure of the divisome.

Studies on division proteins' interactions in various bacterial species, such as E. coli, B. subtilis, S. pneumoniae and M. tuberculosis (Tormo et al., 1986; Wang et al., 1997; Ma \& Margolin, 1999; Haney et al., 2001; Datta et al., 2002, 2006; Haeusser et al., 2004; Lara et al., 2005; Fadda et al., 2007) indicate that they engage in many interactions between each other. These results, however, refer to sporadic tests of a few interactions performed in the species reported above, whereas the only whole interaction web currently available is that of E. coli (Di Lallo et al., 2003; Karimova et al., 2005; D'Ulisse et al., 2007).

It is now generally accepted that knowledge on interactions conserved in other organisms, or 'interologs' (Walhout et al., 2000) ought to represent useful information that will allow the formulation and testing of biological hypotheses (Walhout et al., 2000; Wojcik \& Schachter, 2001). On this basis, in this work we identified the interaction web amongst 11 division proteins of S. pneumoniae in their 66 possible pairwise combinations and compared the results obtained with those for E. coli.

The results showed the existence of a close net constituted of at least 37 homo- and/or heterodimeric protein associations in which each protein generally interacts with more partners, up to 10 for FtsL and FtsZ, whereas the mean number of partners is two or three. PBP1A is the only case described in our results in which the protein interacts with a single partner, i.e. FtsL.

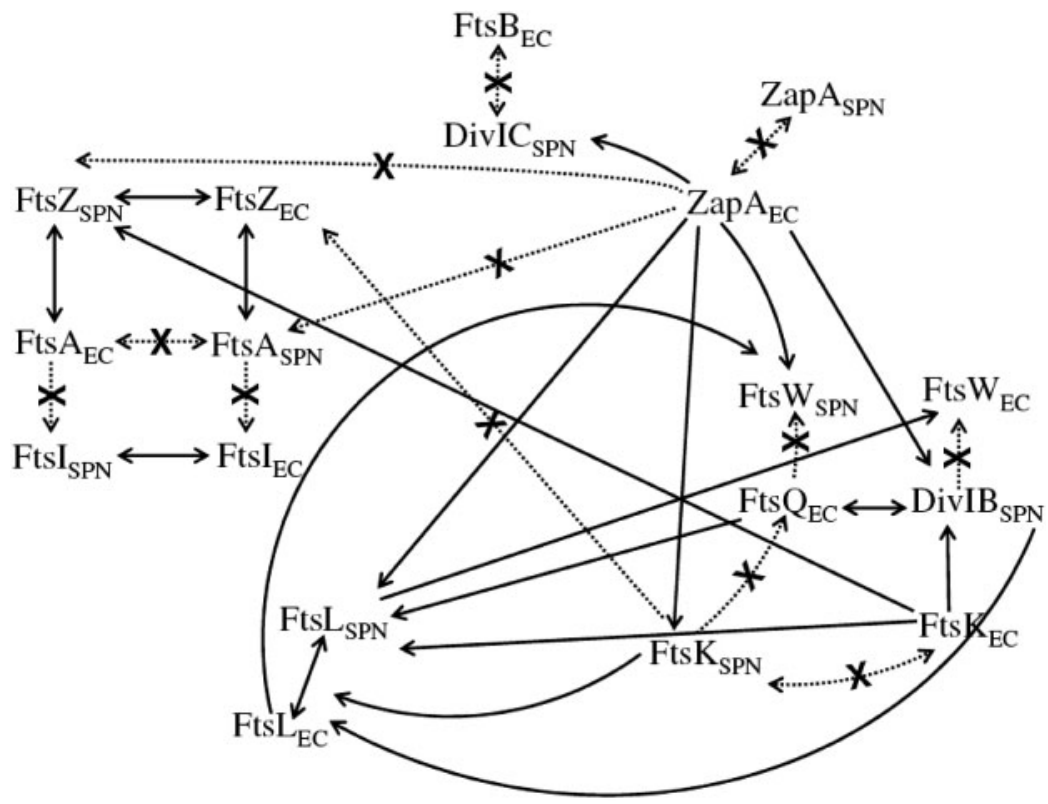

Fig. 3. Cross-interaction between selected cell division protein pairs from $S$. pneumoniae and $E$. coli. The unbroken arrows indicate the presence of an interaction between species, while dotted arrows that are crossed out indicate the absence of interaction. In most cases, we observed interaction with both the $434-X_{E C} / P 22-Y_{S P N}$ and the 434- $X_{S P N} / P 22-$ $Y_{E C}$ pairs. Only the interaction between FtsK $_{E C}-F_{t s Z_{S P N}}$ and FtsK $_{E C}-D_{\text {DivlB }}$ SPN were observed unidirectionally. Cross-interactions between ZapA and the other division proteins were tested only with the $434-Z a p A_{E C}$ and P22-Y S. pneumoniae partners. 
The comparison of this network with the one already established for E. coli (Di Lallo et al., 2003) showed, for the nine common proteins, that among these 45 possible pairs of proteins, 30 show an analogous pattern in both microorganisms: 23 interact and seven do not. The similarity of these two interactomes is not surprising since various 'comparative proteomics' strategies applied to the search for pairs of potential orthologues of known interacting protein partners in Saccharomyces cerevisiae have been performed to identify potentially conserved interactions, or 'interologs' (Walhout et al., 2000), in Caenorhabditis elegans. At least $16 \%$ of the protein interactions in these networks are indeed conserved.

Our data raise some considerations: (i) the maintenance (conservation) of analogous interactomes between two phylogenetically distant species could be indicative of the biological significance of each interaction between the protein and its partners; (ii) the presence of interaction overlaps in the two networks suggests the existence of a common core of divisome proteins; (iii) if this interpretation is correct we should expect to find cross-interactions among S. pneumoniae and E. coli division proteins.

This hypothesis is supported both by the results shown in Fig. 3 and by the observation of Wang et al. (1997) of heterologous interactions between division proteins (FtsZ and FtsA) of B. subtilis and E. coli. Notably, the crossinteractions described here were observed between proteins of two bacterial species with a discrete sequence homology. The percentage of identical amino acid residues determined by Lipman-Pearson protein alignment between $S$. pneumoniae and E. coli proteins FtsA, FtsZ, DivIB and PBP2x (FtsI) is 33.0, 54.7, 17.1 and $24.1 \%$, respectively (Massidda et al., 1998).

The cross-interaction ability described in this paper may reflect a functional inter-exchangability from one organism to another. This is the case for S. pneumoniae DivIB protein, which phenotypically suppresses the defects of an E. coli FtsQ null mutant (D'Ulisse et al., 2007). Heterologous complementation among division functions of phylogenetically distant bacteria have also been described by other authors (Ma et al., 1997; Gaikwad et al., 2000; Momynaliev et al., 2002; Dziadek et al., 2003; Osawa \& Erickson, 2006). We are presently performing other complementation assays to establish whether other $S$. pneumoniae division proteins can substitute for their E. coli orthologues.

It is generally admitted that various levels of prudence need to be considered when analysing the results obtained by THA. This last notion comes from the application of the Saccharomyces cerevisiae THA, the first and most widely used tool in protein-protein interaction. This assay often highlights false positive or negative interactions. False positives are due to the overexpression of the reporter protein fusions, which could result in magnifying potentially weak interactions whereas the false negatives could be due, for example, to low expression levels and/or instability of the molecules tested. Presently, we have no data that allow evaluation of the limit of the THA used in this work. However, although the number of interactions tested with the phage THA is very low compared with that obtained with the yeast THA, some data suggest that our system excludes the false (or indirect) interactions: formally, if a protein $\mathrm{X}$ interacts with both $\mathrm{A}$ and $\mathrm{B}$, false positives could be due to indirect interaction between $A$ and $B$ mediated by $\mathrm{X}$ in a complex such as $\mathrm{A}-\mathrm{X}-\mathrm{B}$. Besides the examples reported in previous papers (Di Lallo et al., 2003; D'Ulisse et al., 2007), the absence of interaction between ZipA and FtsA in E. coli suggests that the bridge eventually formed with FtsZ, which interacts with both proteins, is not revealed. Analogously, the assay does not reveal interactions between the E. coli proteins FtsZ-FtsW and FtsZ-FtsB although all of them also interact with ZapA. In addition, the interactions described in this paper were observed in a heterologous system, $S$. pneumoniae proteins tested in $E$. coli. The formation of false positives should need an E. coli 'bridge' protein able to interact with both the $S$. pneumoniae partners. Other kinds of false positives are, however, possible, such as the one due to the overexpression of proteins magnifying potentially weak interactions and leading to false signals. In these cases, levels of residual $\beta$-galactosidase synthesis close to $50 \%$ could represent weak or transient interactions amplified by protein overexpression. In any case, the $S$. pneumoniae species-specific interactions have already been confirmed by co-immunoprecipitation, similarly to previous reports (D'Ulisse et al., 2007; Fadda et al., 2007). Finally, as far as false negatives are concerned, this aspect can be related to the expression level and/or stability of the proteins under investigation. Generally, it is easily solved by observing whether there are two molecules, $\mathrm{X}$ and $\mathrm{Y}$, that do not interact with each other, but each of which is able to interact with other partners; thus the lack of interaction between them is real. This condition was verified for 29 protein pairs that did not shown any interaction between them.

In conclusion, the comparative analysis of the division protein interactomes reported in this work allows the identification of protein-protein interaction representatives of a minimal common divisome in Bacteria. For this reason, these results may be helpful for both a deeper comprehension of some aspects related to bacterial divisome formation and the elucidation of the cell division process in the Gram-positive coccus S. pneumoniae, where some genetic tools are as yet underused. Finally, given the importance of the cell division proteins as primary targets for the development of new broad-spectrum antibacterial inhibitors, the knowledge of the conserved protein-protein interactions, between phylogenetically distant species, should reinforce their attractiveness in this respect.

\section{ACKNOWLEDGEMENTS}

We thank André Zapun and Thierry Vernet, Institut de Biologie Structurale, CNRS Grenoble, for providing polyclonal antibodies. We 
also thank Valeria D’Ulisse for her help in performing interaction experiments.

\section{REFERENCES}

Ayala, J. A., Garrido, T., Pedro, M. A. \& Vicente, M. (1994). Molecular biology of bacterial septation. In Bacterial Cell Wall, pp. 73-101. Edited by J. M. Ghuysen \& R. Hackenbeck. Amsterdam: Elsevier Science BV.

Buddelmeijer, N. M. \& Beckwith, J. (2004). A complex of the Escherichia coli cell division proteins FtsL, FtsB and FtsQ forms independently of its localization to the septal region. Mol Microbiol 52, 1315-1327.

Datta, P., Dasgupta, A., Bhakta, S. \& Basu, J. (2002). Interaction between FtsZ and FtsW of Mycobacterium tuberculosis. J Biol Chem 277, 24983-24987.

Datta, P., Dasgupta, A., Singh, A. K., Mukheriee, P., Kundu, M. \& Basu, J. (2006). Interaction between FtsW and penicillin-binding protein 3 (PBP3) directs PBP3 to mid-cell, controls cell septation and mediates the formation of a trimeric complex involving FtsZ, FtsW and PBP3 in mycobacteria. Mol Microbiol 62, 1655-1673.

Di Lallo, G., Castagnoli, L., Ghelardini, P. \& Paolozzi, L. (2001). A two-hybrid system based on chimeric operator recognition for studying protein homo/heterodimerization in Escherichia coli. Microbiology 147, 1651-1656.

Di Lallo, G.,, Fagioli, M., Barionovi, D., Ghelardini, P. \& Paolozzi, L. (2003). Use of a two-hybrid assay to study the assembly of a complex multicomponent protein machinery: bacterial septosome differentiation. Microbiology 149, 3353-3359.

D’Ulisse, V., Fagioli, M., Ghelardini, P. \& Paolozzi, L. (2007). Three functional subdomains of the Escherichia coli FtsQ protein are involved in its interaction with the other division proteins. Microbiology 153, 124-138.

Duong, F. \& Wickner, W. (1997). Distinct catalytic roles of the SecYE, SecG and SecDFyajC subunits of preprotein translocase holoenzyme. EMBO J 16, 2756-2768.

Dziadek, J., Rutherford, S. A., Madiraju, M. V., Atkinson, M. A. \& Rajagopalan, M. (2003). Conditional expression of Mycobacterium smegmatis fts $Z$, an essential cell division gene. Microbiology 149, 1593-1603.

Erickson, H. P. (2007). Evolution of the cytoskeleton. Bioessays 29, 668-677.

Errington, J., Daniel, R. A. \& Scheffers, D. J. (2003). Cytokinesis in bacteria. Microbiol Mol Biol Rev 67, 52-65.

Fadda, D., Santona, A., D'Ulisse, V., Ghelardini, P., Ennas, M. G., Whalen, M. B. \& Massidda, O. (2007). Streptococcus pneumoniae DivIVA: localization and interactions in a MinCD-free context. $J$ Bacteriol 189, 1288-1298.

Gaikwad, A., Babbarwal, V., Pant, V. \& Mukherjee, S. K. (2000). Pea chloroplast FtsZ can form multimers and correct the thermosensitive defect of an Escherichia coli ftsZ mutant. Mol Gen Genet 263, 213-221.

Goehring, N. W. \& Beckwith, J. (2005). Diverse paths to midcell: assembly of the bacterial cell division machinery. Curr Biol 15, R514R526.

Goehring, N. W., Gonzalez, M. D. \& Beckwith, J. (2006). Premature targeting of cell division proteins to midcell reveals hierarchies of protein interactions involved in divisome assembly. Mol Microbiol 61, 33-45.

Haeusser, D. P., Schwartz, R. L., Smith, A. M., Oates, M. E. \& Levin, P. A. (2004). EzrA prevents aberrant cell division by modulating assembly of the cytoskeletal protein FtsZ. Mol Microbiol 52, 801-814.
Haney, S. A., Glasfeld, E., Hale, C., Keeney, D., He, Z. \& de Boer, P. (2001). Genetic analysis of the Escherichia coli FtsZ-ZipA interaction in the yeast two-hybrid system. Characterization of FtsZ residues essential for the interactions with ZipA and with FtsA. J Biol Chem 276, 11980-11987.

Harry, E., Monahan, L. \& Thompson, L. (2006). Bacterial cell division: the mechanism and its precision. Int Rev Cytol 253, 27-94.

Karimova, G., Dautin, N. \& Ladant, D. (2005). Interaction network among Escherichia coli membrane proteins involved in cell division as revealed by bacterial two-hybrid analysis. J Bacteriol 187, 2233-2243.

Laemmli, U. K. (1970). Cleavage of structural proteins during the assembly of the head of bacteriophage T4. Nature 227, 680-685.

Lara, B., Rico, A. I., Petruzzelli, S., Santona, A., Dumas, J., Biton, J., Vicente, M., Mingorance, J. \& Massidda, O. (2005). Cell division in cocci: localization and properties of the Streptococcus pneumoniae FtsA protein. Mol Microbiol 55, 699-711.

Ma, X. \& Margolin, W. (1999). Genetic and functional analyses of the conserved C-terminal core domain of Escherichia coli FtsZ. J Bacteriol 181, 7531-7544.

Ma, X., Sun, Q., Wang, R., Singh, G., Jonietz, E. L. \& Margolin, W. (1997). Interactions between heterologous FtsA and FtsZ proteins at the FtsZ ring. J Bacteriol 179, 6788-6797.

Margolin, W. (2000). Themes and variations in prokaryotic cell division. FEMS Microbiol Rev 24, 531-548.

Margolin, W. (2003). Bacterial division: the fellowship of the ring. Curr Biol 13, R16-R18.

Massidda, O., Anderluzzi, D., Friedli, L. \& Feger, G. (1998). Unconventional organization of the division and cell wall gene cluster of Streptococcus pneumoniae. Microbiology 144, 3069-3078.

Miller, J. H. (1972). Experiments in Molecular Genetics. Cold Spring Harbor, NY: Cold Spring Harbor Laboratory.

Momynaliev, K. T., Smirnova, O. V., Lazyrev, V. N., Akopian, T. A., Chelysheva, V. V., Ayala, J. A., Simankova, A. N., Borchsenius, S. N. \& Govorun, V. M. (2002). Characterization of the Mycoplasma hominis $\mathrm{fts} Z$ gene and its sequence variability in mycoplasma clinical isolates. Biochem Biophys Res Commun 293, 155-162.

Morlot, C., Zapun, A., Dideberg, O. \& Vernet, T. (2003). Growth and division of Streptococcus pneumoniae: localization of the high molecular weight penicillin-binding proteins during the cell cycle. Mol Microbiol 50, 845-855.

Morlot, C., Noirclerc-Savoye, M., Zapun, A., Dideberg, O. \& Vernet, T. (2004). The D,D-carboxypeptidase PBP3 organizes the division process of Streptococcus pneumoniae. Mol Microbiol 51, 1641-1648.

Noirclerc-Savoye, M., Le Gouellec, A., Morlot, C., Dideberg, O., Vernet, T. \& Zapun, A. (2005). In vitro reconstitution of a trimeric complex of DivIB, DivIC and FtsL, and their transient co-localization at the division site in Streptococcus pneumoniae. Mol Microbiol 55, 413-424.

Osawa, M. \& Erickson, H. P. (2006). FtsZ from divergent foreign bacteria can function for cell division in Escherichia coli. J Bacteriol 188, 7132-7140.

Pinho, M. G. \& Errington, J. (2005). Recruitment of penicillin-binding protein PBP2 to the division site of Staphylococcus aureus is dependent on its transpeptidation substrates. Mol Microbiol 55, 799-807.

Sambrook, J., Fritsch, E. F. \& Maniatis, T. (1989). Molecular Cloning: a Laboratory Manual. Cold Spring Harbor, NY: Cold Spring Harbor Laboratory.

Tamames, J., González-Moreno, M., Mingorance, J., Valencia, A. \& Vicente, M. (2001). Bringing gene order into bacterial shape. Trends Genet 17, 124-126. 
Tormo, A., Ayala, J. A., de Pedro, M. A., Aldea, M. \& Vicente, M. (1986). Interaction of FtsA and PBP3 proteins in the Escherichia coli septum. J Bacteriol 166, 985-992.

Towbin, H., Staehelin, T. \& Gordon, J. (1979). Electrophoretic transfer of proteins from acrylamide gels to nitrocellulose sheets: procedure and some applications. Proc Natl Acad Sci U S A 76, 4350-4354.

Vicente, M. \& Errington, J. (1996). Structure, function and controls in microbial division. Mol Microbiol 20, 1-7.

Vicente, M. \& Rico, A. I. (2006). The order of the ring: assembly of Escherichia coli cell division components. Mol Microbiol 61, 5-8.

Vicente, M., Rico, A. I., Martínez-Arteaga, R. \& Mingorance, J. (2006). Septum enlightenment: assembly of bacterial division proteins. $J$ Bacteriol 188, 19-27.

Walhout, A. J., Sordella, R., Lu, X., Hartley, J. L., Temple, G. F., Brasch, M. A., Thierry-Mieg, N. \& Vidal, M. (2000). Protein interaction mapping in C. elegans using proteins involved in vulval development. Science 287, 116-122.
Wang, X., Huang, J., Mukherjee, A., Cao, C. \& Lutkenhaus, J. (1997). Analysis of the interaction of FtsZ with itself, GTP, and FtsA. $J$ Bacteriol 179, 5551-5559.

Weiss, D. S. (2004). Bacterial cell division and the septal ring. Mol Microbiol 54, 588-597.

Wojcik, J. \& Schachter, V. (2001). Protein-protein interaction map inference using interacting domain profile pairs. Bioinformatics 17 (Suppl. 1), S296-S305.

Yura, T., Mori, H., Nagai, H., Nagata, T., Ishihama, A., Fujita, N., Isono, K., Mizobuchi, K. \& Nakata, A. (1992). Systematic sequencing of the Escherichia coli genome: analysis of the 0-2.4 min region. Nucleic Acids Res 20, 3305-3308.

Zapun, A., Vernet, T. \& Pinho, M. G. (2008). The different shapes of cocci. FEMS Microbiol Rev 32, 345-360.

Edited by: W. Margolin 